\title{
Implementing Computer Aided Material Design of Multiple Cursors for Disabled People with InLinPreRa
}

\author{
Ching-Tien Shih", a, Shu-Chen $\mathrm{Hsu}^{2,3, \mathrm{~b}}$ \\ 'Department of Digital Game \& Animation Design (DGAD), Tung Fang Design Institute, \\ Kaohsiung City 82941, Taiwan (R.O.C) \\ ${ }^{2}$ Kaosiung municipal Nanzih Special School, Kaohsiung, Taiwan \\ ${ }^{3}$ Department of Marketing Distribution Management, Kao Yuan University \\ actshih@mail.tf.edu.tw, bdemi8468@hotmail.com
}

Keywords: Assistive technology, Mouse, Cursor, Computer Aided Material Design, Happiness technology, InLinPreRa.

\begin{abstract}
Through adjusting the curriculum, comprehensive learning environment design, and information technology aids to effectively integrate disabled students with learning, in order to assist them to control environment or communicate with people, and to improve their ability of independent living. Orange technology is the current trend to advocate that high-tech should be emphasized on human and social care, and to create happiness technology. However, whether these various assistive devices and the blueprint of living and teaching can bring the true happiness to disabled students needs to be considered. Therefore, this project proposes a design of single mouse with multiple cursors to assist these disabled students who have poor control of hand movements or poor hand-eye coordination, and who have difficult to move the cursor to click or enter commands and data. This design is able disabled students to shorten the computer operating time through quickly moving the mouse cursor to the position, and the experimental teaching is also carried out. Not only to analyze the effectiveness of the proposed design, but also to realize whether the design is effectively integrated into the comprehensive learning environment of special students under the experimental teaching process, and whether the design is able to improve disabled students' successful learning experience and to enhance their curriculum participation and happiness.
\end{abstract}

\section{Introduction}

Computer technologies have an important role to play in promoting success in employment for disabled people. They can use computer technology to increase their daily living tasks by individuals with mental retardation and independence in the performance of vocational. It is a great opportunity to broaden their lives. With the aid of computer technologies, such individuals can increase their independence in their performance of both vocational and daily living tasks and have more opportunities to broaden their lives and raise their overall level of competency [1-8].

For people with disabilities, assistive input devices are designed to provide them additional accessibility to use computer, and enable them to use simple behavior actions to operate computer (such as hand/foot movement or swinging, thumb/finger poking) [9]. However, most computer input devices (i.e., mouse, keyboard) are designed for mainstream people [10-13]. People with disabilities may encounter difficulties using a standard computer input device, particularly if the disability is severe, such as with a high-level cervical spinal cord injury (SCI) [14, 15]. Various computer assistive input devices have been proposed to comply with the needs of people with disabilities [8, 16-18]

Therefore, it is important issue to select and design a single mouse with multiple cursors for disabled people. This study applies the Incomplete Linguistic Preference Relations (InLinPreRa) analytical framework to measuring the satisfaction of single mouse with multiple cursors for disabled people implementation as well as to verify the feasibility of this proposed approach. This model not only serves as a checking mechanism, but also helps in analyzing the organizational 
ability by considering key success factors of single mouse with multiple cursors for disabled people implementation.

\section{Literature Review}

$\mathrm{Xu}$ [19] proposed the Incomplete Linguistic Preference Relations, during the process of pairwise comparison, each expert can select anyone of the explicit items as the standard according to his/her preference or recognition, and then the pairwise comparison would be carried out between the adjoining items in order obtain the original preference matrix; complete linguistic preference relation counters the fact that all of the attribute decision-making experts carry out the pairwise comparison through preference matrix; when the decision maker uses pairwise comparison to compare the original preference values, and the remaining unknown variables add with the adjoining numbers that equals to 0 through the corresponding opposite numbers so as to obtain the complete matrix, this is called Incomplete Linguistic Preference Relations. Linguistic preference relations are usually used by decision makers to express their linguistic preference information based on pairwise comparisons [19, 20]. The Decision Making Matrix of Incomplete Linguistic Preference Relations definitions are described as follows:

Definition 1: Incomplete Linguistic Preference Additive Relation. Let $A=\left(a_{i j}\right)_{n \times n}$ be linguistic preference relation, if $A$ is an incomplete linguistic preference relation, it counters the fact that decision makers can carry out pairwise comparison for attributes so as to satisfy Eq. 1.

$$
a_{i j} \in S, a_{i j} \oplus a_{j i}=S_{0}, a_{i i}=S_{0}
$$

Definition 2: Incomplete Linguistic Consistent Additive Preference Relation. Let $A=\left(a_{i j}\right)_{n \times n}$ be complete consistent additive preference relation, which counters all of the $i, j, k$ decision makers for pairwise comparison, if $a_{i k}>S_{0}$ represents $x_{i}$ is better than $x_{k}$; while $a_{k j}>S_{0}$ represents $x_{k}$ is better than $x_{j}$, then $a_{i j}>S_{0}$ can be derived the equation of $x_{i}$ better than $x_{j}$ is

$$
a_{i j}=a_{i k} \oplus a_{k j}
$$

\section{Framework for Implementing Computer Aided Material Design of Multiple Cursors for Disabled People with InLinPreRa}

Investigating the influential factors on single mouse with multiple cursors for disabled people implementation. The influential factors are derived though widespread investigation and consultation with several experts. There are (C1) Expansion (C2) Stability of Device, (C3), easy to maintain (C4) Compatible with computer, (C5) easy to operate, (C6) User-friendly interface (C7) Budget.

Determining the Priority Weights of Influential Factors. Subjectivity and vagueness within the measuring process are dealt with using linguistic variables quantified in a scale of ${ }^{[-t, t]}$. This study is used linguistic to express their strength of preference among influential factor.

Linguistic Variables. This study provides the evaluators simple linguistic terms quantified on a scale of $[-8,8]$ to express their strength of preference among influential factors (see Table 1). Linguistic variables [8] are simultaneously used to measure the likelihood of success/failure regarding each influential factor (see Table 2).

Table 1. Linguistic terms for the importance weights of influential factors.

\begin{tabular}{cccc}
\hline Definition & value & value \\
\hline Absolutely more important (AB) & 8 & Less Weakly more important (LWK) & -2 \\
Very strongly more important (VS) & 6 & Less Strongly more important (LST) & -4 \\
Strongly more important (ST) & 4 & Less Very strongly more important (LVS) & -6 \\
Weakly more important (WK) & 2 & Less Absolutely more important (LAB) & -8 \\
Equally important (EQ) & 0 & & \\
\hline
\end{tabular}


Table 2. Linguistic variables for the priority ratings of possible outcome.

\begin{tabular}{cc}
\hline Definition & value \\
\hline Very high $(\mathrm{VH})$ & 4 \\
High $(\mathrm{H})$ & 2 \\
Fair $(\mathrm{F})$ & 0 \\
Less High $(\mathrm{LH})$ & -2 \\
Less Very high LVH) & -4 \\
\hline
\end{tabular}

Obtaining Priority Weights of Influential Factor. Construct pairwise comparison matrices amongst the influential factors $\left(\mathrm{C}_{r}, r=1,2, \ldots, k\right)$. The evaluators $\left(\mathrm{E}_{e}, e=1,2, \ldots, n\right)$ used three types of pairwise comparisons algorithm which are horizontal vertical and oblique to construct pairwise comparison matrices. Used the horizontal comparison of matrices are as below:

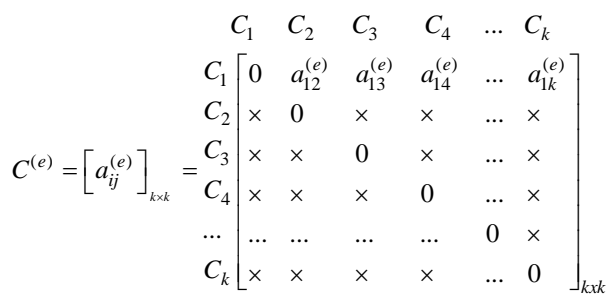

The remaining $a_{i j}^{(e)}$ can be calculates using Eqs.(1) and Eqs.(2) to obtain the other known $\times$ of triangular second half. Finally, obtain the full preference matrix.

Transform the preference value $a_{i j}^{(e)}$ into $b_{i j}^{(e)}$ in an interval scale [0,1], then the matrix $C t$ obtained as $C t=f\left(C^{(e)}\right)$. The transformation function is given by

$$
\begin{aligned}
& f:[-a, a] \rightarrow[0,1], f(x)=\frac{x+a}{2 a}
\end{aligned}
$$

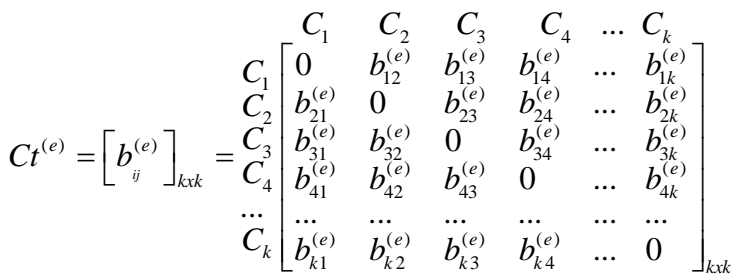

Utilize the method of average value to integrate the judgment values of $n$ evaluators, namely

$\bar{C}=\left[p_{i j}\right]_{k x k}$

$$
\begin{gathered}
p_{i j}=\frac{1}{n}\left(b_{i j}^{(1)}+b_{i j}^{(2)}+\ldots+b_{i j}^{(n)}\right)=\frac{1}{n} \sum_{e=1}^{n} b^{(e)} \\
i=1,2, \ldots, k \quad, \quad j=1,2, \ldots, k
\end{gathered}
$$

Use $h_{i j}$ to indicate the normalized preference values of each influential factor, such as

$$
\begin{aligned}
& \begin{array}{llllll}
C_{1} & C_{2} & C_{3} & C_{4} & \ldots & C_{k}
\end{array} \\
& \left.\overline{\bar{C}=\left[h_{i j}\right]_{k x k}=} \begin{array}{r}
C_{1} \\
C_{2}
\end{array} \begin{array}{lllllll}
0 & C_{12}^{(e)} & h_{13}^{(e)} & h_{14}^{(e)} & \ldots & h_{1 k}^{(e)} \\
C_{4}(e) & 0 & h_{23}^{(e)} & h_{24}^{(e)} & \ldots & h_{2 k}^{(e)} \\
C_{4} & h_{31}^{(e)} & h_{32}^{(e)} & 0 & h_{34}^{(e)} & \ldots & h_{3 k}^{(e)} \\
& h_{41}^{(e)} & h_{42}^{(e)} & h_{43}^{(e)} & 0 & \ldots & h_{4 k}^{(e)} \\
\ldots & \ldots & \ldots & \ldots & \ldots & \ldots \\
C_{k} & h_{k 1}^{(e)} & h_{k 2}^{(e)} & h_{k 3}^{(e)} & h_{k 4}^{(e)} & \ldots & 0
\end{array}\right]_{k x k}
\end{aligned}
$$




$$
h_{i j}=\frac{p_{i j}}{\sum_{i=1}^{k} p_{i j}} \quad i=1,2, \ldots k, j=1,2, \ldots, k
$$

Given the ${ }^{r} w$ denoting the priority weight of influential factor $r$, the priority weight of each factor can be obtained that is

$$
\begin{aligned}
& { }^{r} w=\frac{\sum_{j=1}^{k} h_{r j}}{\sum_{i=1}^{k} \sum_{j=1}^{k} h_{i j}} \quad i=1,2, \ldots, k^{r} w=\frac{\sum_{j=1}^{k} h_{r j}}{\sum_{i=1}^{k} \sum_{j=1}^{k} h_{i j}} i=1,2, \ldots, k \\
& { }^{1} w,{ }^{2} w, \ldots,{ }^{k} w, \quad{ }^{r} w \in[0,1], \quad \sum_{r=1}^{k}{ }^{r} w=1
\end{aligned}
$$

Determining the Priority Ratings for Possible Outcome Regarding Factors. The evaluators are asked to express their subjective judgments regarding the preference ratings of possible outcome $\left(A_{i}, i=1,2, \ldots, m\right)$ regarding each influential factor in linguistic terms, as listed in Table 2.

The evaluators used three types of pairwise comparisons algorithm to choose the better of two possible outcomes for a set of $m-1$ preference data under each influential factor. Uses the horizontal comparison kinds of matrices are below.

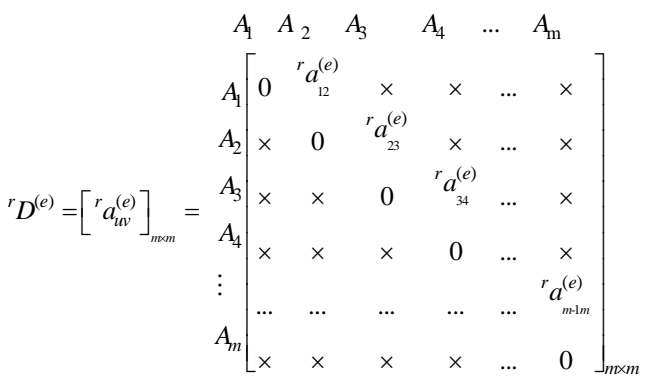

Using Eqs. (1) and Eqs. (2) to obtain the corresponding value. Finally, obtain the full preference matrix.

Next, the preference value ${ }^{r} a_{u v}^{(e)}$ is transformed in the range [-4, 4] into ${ }^{r} b_{u v}^{(e)}$ in an interval scale $[0,1]$, then the matrix ${ }^{r} D t$ obtained as ${ }^{r} D t=f\left({ }^{r} D^{(e)}\right)$. The transformation function is given by

$$
\begin{aligned}
& f:[-a, a] \rightarrow[0,1], f(x)=\frac{x+a}{2 a} \\
& { }^{r} D t=\left[{ }^{r} b_{u v}^{(e)}\right]_{m x m} u, v=1,2, \ldots, m
\end{aligned}
$$

Utilize the method of average value to integrate the judgment values of $n$ evaluators, namely

$$
\begin{aligned}
& r \bar{D}=\left[q_{u v}\right]_{m x m} \\
& q_{u v}=\frac{1}{n}\left({ }^{r} b_{u v}^{(1)}+{ }^{r} b_{u v}^{(2)}+\ldots+{ }^{r} b_{u v}^{(e)}\right) \\
& \quad=\frac{1}{n} \sum_{e=1}^{n} b_{u v}^{(e)} \quad u=1,2, \ldots, m, \quad v=1,2, \ldots, m
\end{aligned}
$$

Use ${ }^{r} \lambda_{u v}$ to indicate the normalized preference values of each influential factor, such as

$$
\begin{aligned}
& { }^{r} \overline{\bar{D}}=\left[{ }^{r} \lambda_{u v}\right]_{m x m} \\
& { }^{r} \lambda_{u v}=\frac{q_{u v}}{\sum_{u=1}^{m} q_{u v}} u, v=1,2, \ldots, m
\end{aligned}
$$


Consequently, ${ }^{r} \varphi_{u}$ denoting the average rating of possible outcome $u$ with respect to influential factor $r$ is provided. The desired rating of each possible outcome can be obtained for each influential factor that is

$$
{ }^{r} \varphi_{u}=\frac{1}{\pi} \sum_{v=1}^{m}{ }^{r} \lambda_{u v}
$$

Obtaining the Priority Weight for Prediction. Multiplying the priority weights of influential factors by the ratings of possible outcomes, a predicted value $Z_{u}$ for chance in success/failure implementation is obtained as:

$$
Z_{u}={ }^{r} \varphi_{u} \otimes{ }^{r} w
$$

\section{Empirical Case Study}

We have a meeting with all members to make sure they know what the model meant and how to measure the importance weights of influential factors before prediction. Seven major influential factors are considered in this problem of measuring the possibility of happiness single mouse with multiple cursors for disabled people implementation. The sequences of these seven influential factors are randomly assigned, namely. The seven major risk factors are (C1) Expansion (C2) Stability of Device, (C3), easy to maintain (C4) Compatible with computer, (C5) easy to operate, (C6) User-friendly interface (C7) Budget.

The priority weight of each influential factor can to be obtained by Framework. The priority weight and rank of each influential factor assessed by eleven evaluators are listed as below:.

$C_{6}(0.166)>C_{2}(0.163)>C_{7}(0.159)>C_{3}(0.144)>C_{5}(0.126)>C_{4}(0.123)>C_{2}(0.120)$

The results demonstrate that the five most important influential factors are (C6) User-friendly interface (0.166) (C7) Budget (0.163), (C2) Stability of Device (0.159), (C3), easy to maintain (0.144), (C5) easy to operate (0.126).

The prediction weight for single mouse with multiple cursors for disabled people system implementation is calculated as follow:

$$
\begin{aligned}
& Z_{\text {success }}=\left(0.647 \times 0.126+0.569 \times 0.120+0.534 \times 0.144+Z_{\text {failure }}=(0.353 \times 0.126+0.431 \times 0.120+0.466 \times 0.144+\right. \\
& 0.685 \times 0.163+0.569 \times 0.159+0.710 \times 0.166+\quad 0.315 \times 0.163+0.431 \times 0.159+0.710 \times 0.166+ \\
& 0.655 \times 0.123)=0.627 \\
& 0.345 \times 0.123)=0.373
\end{aligned}
$$

\section{Conclusion and Contribution}

This study used the Incomplete Linguistic Preference Relations (InLinPreRa) analytical framework to implementing computer aided material design of multiple cursors for disabled people as well as to verify the feasibility of this proposed approach. Experiments show the success possibility of implementing single mouse with multiple cursors for disabled people over 0.6 . The whole evaluation shows that "User-friendly interface" is the most important influential factor. The proposed approach is based on incomplete linguistic preference relations, rather than using conventional multiplicative preference relation. Based on the operational laws of the linguistic evaluation scale, we develop a simple and practical method for constructing a consistent complete linguistic preference relation by using the additive transitivity property. This study not only shortens the decision time and makes sufficiently using of the provided preference information, but also maintains the decision maker's consistency level and avoids checking the consistency of linguistic. Namely, this method considers only $n-1$ judgments, whereas the traditional analytic hierarchy approach (that is AHP or FAHP) uses $n(n-1) / 2$ judgments in a preference matrix with $n$ attributes or alternatives. It is clear that the proposed approach is faster to execute and more efficient than the conventional analytic hierarchy methodologies. We hope that the result of the study can provide disabled people to select and design a suitable single mouse with multiple cursors. 


\section{Acknowledgements}

The authors would like to thank the National Science Council of the Republic of China, Taiwan for financially supporting this research under Project 104-2511-S-272 -001 -

\section{References}

[1] D. K. Davies, S. E. Stock, M. L. Wehmeyer, Enhancing independent time-management skills of individuals with mental retardation using a palmtop personal computer, Mental Retard. 40(5) (2002) 358-365.

[2] J. Bache, G. Derwent, Access to computer-based leisure for individuals with profound disabilities, Neuro Rehabilitation, 23(4) (2008) 343-350.

[3] V. Bernard-Opitz, N. Sriram, S. Nakhoda-Sapuan, Enhancing social problem solving in children with autism and normal children through computer-assisted instruction, J. Autism Development. Disord. 31(4) (2001) 377-384.

[4] B. S. Hoppestad, Essential elements for assessment of persons with severe neurological impairments for computer access utilizing assistive technology devices: A Delphi study, Disabil. Rehabil. Assist. Technol. 1(1-2) (2006) 3-16.

[5] D. K. Davies, S. E. Stock, M. L. Wehmeyer, Enhancing independent task performance for individuals with mental retardation through use of a handheld self-directed visual and audio prompting system, Educ. Train. Mental Retard. Development. Disabil. 37(2) (2002) 209-218.

[6] H. Ritchie, P. Blanck, The promise of the Internet for disability: a study of on-line services and web site accessibility at Centers for Independent Living, Behav. Sci. Law, 21(1) (2003) 5-26.

[7] S. Burgstahler, The role of technology in preparing youth with disabilities for postsecondary education and employment, J. Special Educ. Technol. 18(4) (2003) 7-20.

[8] C. T. Shih, C. H. Shih, C. H. Luo, Evaluation of a dynamic pointing assistive program in cursor pointing efficiency for people with disabilities, Technol. Disabil. 23(4) (2011) 215-222.

[9] C. H. Shih, C. J. Shih, C. T. Shih, Assisting people with multiple disabilities by actively keeping the head in an upright position with a Nintendo Wii Remote Controller through the control of an environmental stimulation, Res. Development. Disabil. 32(5) (2011) 2005-2010.

[10] J. Abascal, C. Nicolle, Moving towards inclusive design guidelines for socially and ethically aware HCI, Interact. Comput. 17(5) (2005) 484-505.

[11]M. G. Brodwin, T. Star, E. Cardoso, Computer assistive technology for people who have disabilities: Computer adaptations and modifications, J. Rehabil. 70(3) (2004) 28-33.

[12]A. M. Cook, S. M. Hussey, Assistive technologies: Principles and practice: St. Louis, MO: Mosby, Inc., 2002.

[13]C. H. Shih, C. T. Shih, P. H. Pi, Using an Extended Automatic Target Acquisition Program with Dual Cursor technology to assist people with developmental disabilities in improving their pointing efficiency, Res. Development. Disabil. 31(5) (2011) 1506-1513.

[14] S. Fujisawa, K. Ohkubo, T. Nishi, T. Yoshida, Y. Shidama, H. Yamaura, Fundamental research on human interface devices for physically handicapped persons, Industrial Electronics, Control and Instrumentation, 1997. IECON 97. 23rd International Conference on Industrial Electronics, Control, and Instrumentation, IECON Part 3, November 9-14,1997, New Orleans, LA, USA, 1997.

[15]R. S. Rao, R. Seliktar, T. Rahman, Evaluation of an isometric and a position joystick in a target acquisition task for individuals with cerebral palsy, IEEE Trans. Rehabil. Eng. 8(1) (2000) 118-125.. 
[16] Y. L. Chen, W. L. Chen, T. S. Kuo, J. S. Lai, A head movement image (HMI)-controlled computer mouse for people with disabilitiesAnalysis of a time-out protocol and its applications in a single server environment, Disabil. Rehabil. 25(3) (2003) 163-167.

[17] Y. L. Chen, F. T. Tang, W. H. Chang, M. K. Wong, Y. Y. Shih, T. S. Kuo, The new design of an infrared-controlled human-computer interface for the disabled, Rehabil. Eng. IEEE Trans. 7(4) (1999) 474-481.

[18]K. S. Park, K. T. Lee, Eye-controlled human/computer interface using the line-of-sight and the intentional blink, Comput. Ind. Eng. 30 (1996) 463-473.

[19]Z. Xu, Incomplete linguistic preference relations and their fusion, Inf. Fusion, 7(3) (2006) 331337.

[20]Z. Xu, A method for multiple attribute decision making with incomplete weight information in linguistic setting, Knowl. Based Syst. 20(8) (2007) 719-725.

[21]T. C. Wang, S. C. Hsu, Y. C. Chiang, Multi-Criteria Decision Making with Expansion of Incomplete Linguistic Preference Relations, Wseas Trans. Math. 6 (2007) 817-823.

[22] S. C. Hsu, T. C. Wang, Solving multi-criteria decision making with incomplete linguistic preference relations, Expert Syst. Appl. 38(9) (2011) 10882-10888.

[23]T. H. Chang, S. C. Hsu, T. C. Wang, C. Y. Wu, Measuring the success possibility of implementing ERP by utilizing the Incomplete Linguistic Preference Relations, Appl. Soft Comput. 12(5) (2012) 1582-1591. 